\title{
Copeptin: a potential blood biomarker for acute ischemic stroke
}

\author{
Mohammed I. Oraby ${ }^{*^{*} \mathbb{D}}$, Rasha H. Soliman', Rehab M. Abd Elkareem² and Amna I. Mohammed ${ }^{1}$
}

\begin{abstract}
Background: Copeptin is a new blood biomarker for acute ischemic stroke which emerged to assist clinicians with decision-making. Serum copeptin can accurately reflect vasopressin concentration, which plays a role in aggravation of inflammatory responses, ions and neurotransmitters dysfunctions. The objective of this work was to investigate the relation between copeptin level as a blood biomarker and the short-term prognosis of acute ischemic stroke after 3 months. The current study included 45 patients with first ever acute ischemic stroke and 45 healthy volunteers as a control. Clinical evaluation, CT and MRI of the brain, NIHSS on admission, and mRS after 3 months were done for the patients, and all the patients and control were subjected to assessment of serum level of copeptin by ELISA technique.
\end{abstract}

Results: Copeptin level was significantly higher in patients with acute ischemic stroke compared to healthy control subjects ( $p$-value $=0.001)$. Also, copeptin level was significantly higher in patients with severe stroke $(\mathrm{NIHSS}>16)$ than in those with mild-to-moderate stroke (NIHSS 0-15) at presentation and in patients with unfavorable outcome (mRS 3-6) when compared to patients with favorable outcome (mRS 0-2) ( $p$-value $=0.003$ and 0.001 , respectively).

Copeptin level was significantly lower in patients who received thrombolytic therapy with rTPA ( $p$-value $=0.049$ ).

Conclusion: Copeptin has an interesting potential as a new prognostic biomarker for patients with acute ischemic stroke as its level was significantly higher in patients with severe stroke and in patients with unfavorable outcome.

Keywords: Ischemic stroke, Copeptin, Prognostic biomarkers, NIHSS, mRS

\section{Background}

Stroke is one of the main causes of severe morbidity worldwide. Early assessment of disease severity, risk for complications and prognosis is essential for optimized care and proper utilization of available healthcare resources [1].

A variety of novel blood biomarkers are under investigations to assist clinicians with accurate decisionmaking. Arginine-vasopressin (AVP) (also known as antidiuretic hormone), is one of the key hormones of the hypothalamic-pituitary-adrenal axis. Copeptin is the C-terminal part of pro-AVP and composed of 39 amino

\footnotetext{
*Correspondence: Drm_Oraby@med.bsu.edu.eg;Drm_Oraby@hotmail.com ${ }^{1}$ Neurology Department, Beni-Suef University, Beni-Suef 62511, Egypt Full list of author information is available at the end of the article
}

acids, it is secreted together with AVP during processing of the precursor peptide [2].

Currently, there is increasing interest in vasopressin as a potential prognostic biomarker in acute ischemic stroke as it plays a role in aggravation of inflammatory responses, ions and neurotransmitters dysfunctions, cerebral edema and as sequelae, increased intracranial pressure [3].

Compared to cortisol and AVP, copeptin is stable in plasma and serum at room temperature and can be easily measured ex vivo in automated or even manual chemiluminescence assays as a 'shadow' fragment of AVP. Also, copeptin is a useful biomarker in the emergency department setting as the results of the assay can be available within 60 min only [4].

Copeptin can mirror subtle changes in levels of stress better than cortisol. The close connection between 
copeptin levels and the degree of activation of the stress axis is the rationale behind its usefulness as a prognostic biomarker in acute ischemic stroke. Also, copeptin is released directly into the systemic circulation, not into CSF, thus bypassing the blood-brain barrier [1].

The objective of this work was to investigate the relation between copeptin level as a blood biomarker and the short-term prognosis of acute ischemic stroke after 3 months.

\section{Methods}

The current study is a case-control study which included 90 subjects; 45 patients with first ever acute ischemic stroke with symptom onset within $24 \mathrm{~h}$ and below the age of 85 years, and 45 healthy volunteers as a control.

Patients with past history of malignancy or degenerative disease of the nervous system, and patients with hemorrhagic stroke were excluded from the study. Also, patients who have autoimmune diseases with or without immunosuppressive therapy and patients with renal failure were excluded.

This study was approved by the authorized ethical committee, and each participant in the study or one of his first degree family members signed a written informed consent.

The patients included in this study were subjected to the following: clinical assessment-careful history taking, general medical examination including vital signs and cardiac assessment, thorough neurovascular examinations. The patients were classified by The TOAST classification (Trial of Org 10,172 in Acute Stroke Treatment) according to clinical presentation and the results of diagnostic studies and distinguish five subtypes of ischemic stroke; large-artery atherosclerosis, cardio embolism, small-vessel occlusion, stroke of other determined etiology, and stroke of undetermined etiology [5].

The National Institutes of Health Stroke Scale (NIHSS) was used to assess the severity of stroke at presentation [6]. NIHSS score was categorized as mild (score: 1-6), moderate (score: 7-15), or severe (score: 16 and above) [7]. Modified Rankin Scale (mRS) was used to assess the functional outcome on day 90 and the score was categorized as good (mRS 0 to 2; independent) or poor ( $\mathrm{mRS} 3$ to 6; dependent or dead) [8].

Laboratory assessment: patients and control groups were subjected to assessment of the following routine investigations; fasting and post-prandial blood sugar, complete blood count, lipid profile, kidney and liver function tests, serum electrolytes, serum uric acid and ESR). Also, serum level of copeptin was evaluated in both cases and control. In patients, copeptin level was evaluated within the first $24 \mathrm{~h}$ of the symptom onset. Serum copeptin levels were measured by enzyme-linked immunosorbent assays (ELISA) using Stat Fax 2100 Microplate Reader, USA. Blood samples for determination of copeptin were collected at presentation into tubes containing potassium EDT. Then kept 10 min for clotting then centrifuged. Serum was separated and kept under $-80^{\circ} \mathrm{C}$.

Radiological assessment included brain computed tomography $(\mathrm{CT})$ to exclude intracerebral hemorrhage using Toshiba multislice 16 computed tomography, Japan and MRI stroke protocol (T1- and T2-weighted image axial, sagittal and coronal views, fluid-attenuated inversion recovery (FIAIR), diffusion-weighted image (DWI) to assess site, and volume of infarction using the 1.5 Tesla Siemens Scanner Magnetom Aera MRI, Germany.

Infarction volume in DWI was measured using the $\mathrm{A} \times \mathrm{B} \times \mathrm{C} / 2$ formula, where $\mathrm{A}$ is the largest diameter and $\mathrm{B}$ is the perpendicular diameter of the infarction, as measured, and $C$ is the sum of the thicknesses of the slices where the infarction was seen [9].

ECG using standard 12 leads electrocardiogram, echocardiography using vivid S5 machine, China, and carotid duplex using Toshiba machine carotid duplex, Japan, using B mode and Doppler flow were done for all the included patients.

\section{Statistical analysis}

Statistical analysis was done using IBM SPSS version 24 (IBM Corporation, USA, Armonk, New York, 2016). Comparison between groups was done using Chi-square test, independent sample $t$ test and one-way ANOVA test. Pearson correlation was used to test for possible correlations between quantitative variables. $P$ value less than or equal to 0.05 was considered significant. Receiver operating characteristic (ROC) curve analysis was used to assess the prognostic role of copeptin level after acute ischemic stroke.

\section{Results}

The current study is a case-control study conducted on 45 patients diagnosed as acute ischemic stroke within the 1st $24 \mathrm{~h}$ and 45 normal healthy controls. Stroke patients and the controls were matched regarding age and gender (Table 1).

The results of clinical scales for stroke are presented in Table 2. The score of NIHSS at admission ranged from 2 to 28 with a mean of $11.18 \pm 5.1$. The score for mRS after 3 months ranged from $0-5$ with a mean of $3 \pm 1.245$. Stroke outcome assessed by mRS revealed; $21(46.7 \%)$ patients with favorable outcome (mRS 0-2) and $24(53.3 \%)$ patients with unfavorable outcome (mRS 3-6).

In this study, the volume of infarction ranged from 5 to $25 \mathrm{ml}$ in $30(66.7 \%)$ patients, from 26 to $50 \mathrm{ml}$ in 13 
Table 1 Demographic data of the studied population

\begin{tabular}{|c|c|c|c|c|}
\hline & & $\begin{array}{l}\text { Patients with acute ischemic stroke } \\
(n=45)\end{array}$ & $\begin{array}{l}\text { Healthy control } \\
(n=45)\end{array}$ & $p$-value \\
\hline Age $[$ mean $\pm S D]$ & & $55.20 \pm 13.8$ & $51.13 \pm 13.4$ & 0.163 \\
\hline \multirow[t]{2}{*}{ Sex } & Females [n(\%)] & $26(57.8 \%)$ & $30(66.7 \%)$ & 0.257 \\
\hline & Males [n (\%)] & $19(42.2 \%)$ & $15(33.3 \%)$ & \\
\hline
\end{tabular}

Table 2 Results of NIHSS and mRS in 45 acute ischemic stroke patients

\begin{tabular}{llll}
\hline Scale & Score & Number (45) & Percent (\%) \\
\hline NIHSS on admission & Mild (0-6) & 9 & 20 \\
& Moderate (7-15) & 27 & 60 \\
& Severe $(\geq 16)$ & 9 & 20 \\
\multirow{2}{*}{ mRS after 3 months } & Favorable outcome & 21 & 46.7 \\
& $(0-2)$ & & \\
& Unfavorable out- & 24 & 53.3 \\
& come (3-6) & & \\
\hline
\end{tabular}

NIHSS National Institutes of Health Stroke Scale, mRS Modified Rankin Scale

(28.9\%) patients and the volume of infarction was more than $50 \mathrm{ml}$ in 2 (4.4\%) patients. Regarding TOAST classification; 21(46.75) patients had cardio embolic stroke, $13(28.9 \%)$ patients had large-artery atherosclerosis and 11(24.4\%) patients had small artery disease.

Copeptin level was higher in acute ischemic stroke patient when compared to healthy control subjects with a statistically significant difference ( $\mathrm{p}$-value $=0.001$ ). Also, copeptin level was significantly higher in patients with severe stroke (NIHSS $>16)$ than in those with mild-tomoderate stroke (NIHSS 0-15) and in patients with unfavorable outcome (mRS 3-6) when compared to patients with favorable outcome (mRS $0-2)(p-v a l u e=0.003$ and 0.001 , respectively) (Table 3 ).

On the other hand, No statistically significant difference was found between copeptin levels in different acute ischemic stroke subtypes according to TOAST classification $(\mathrm{p}$-value $=0.290)($ Table 4$)$.
We studied the relation between some of the most common modifiable and non-modifiable risk factors of stroke and copeptin level (Table 5). Stroke patients who had hypertension, patients with diabetes mellitus and patients with dyslipidemia showed a statistically significant higher levels of copeptin ( $\mathrm{p}$-value $=0.05,0.024$ and 0.05 , respectively).

Among the stroke patients, 11 patients received thrombolytic therapy with rTPA $(24.4 \%)$ while 34 patients were not eligible to receive rTPA (75.6\%). Copeptin level was significantly lower in patients who received rTPA $(\mathrm{p}$-value $=0.049)($ Table 6$)$.

There were significant positive correlations between each of the following parameters and copeptin level; age of the patients, $(\mathrm{r}=0.212$ and $\mathrm{p}$-value $=0.045)$, BMI $(r=0.272$ and $p$-value $=0.009)$, uric $\operatorname{acid}(r=0.256$ and $\mathrm{p}$-value $=0.015)$ and volume of infarction measured by MRI $(r=0.299$ and $p$-value $=0.004)$. Also, there were significant positive correlations between stroke severity at presentation measured by NIHSS $(r=0.328$ and $\mathrm{p}$-value $=0.002)$, clinical outcome after 3 months measured by $\mathrm{mRS}(\mathrm{r}=0.378$ and $\mathrm{p}$-value $=0.001)$ and serum copeptin level (Table 7).

Table 4 Copeptin level in different stroke subtypes according to TOAST classification

\begin{tabular}{llc}
\hline Stroke subtypes & $\begin{array}{l}\text { Copeptin level }(\mathbf{p g} / \mathbf{m l}) \\
\text { Mean } \pm \text { SD }\end{array}$ & p-value \\
\hline Cardio-embolic $(\mathrm{no}=21)$ & $119.61 \pm 48.8$ & 0.290 \\
Large artery disease $(\mathrm{no}=13)$ & $127.07 \pm 42.8$ & \\
Small artery disease $(\mathrm{no}=11)$ & $107.99 \pm 41.5$ & \\
\hline
\end{tabular}

Table 3 Copeptin level in different clinical situations

\begin{tabular}{llc}
\hline & Number & Copeptin level (pg/ml) \\
& & Mean \pm SD \\
\hline Control group & 45 & $76.51 \pm 32.8$ \\
Stroke patients & 45 & $120.52 \pm 45.7$ \\
Mild-moderate stroke at presentation & 36 & $95.47 \pm 40.3$ \\
Severe stroke at presentation & 9 & $139.45 \pm 38.7$ \\
Stroke patients with favorable outcome & 21 & $93.47 \pm 40.3$ \\
Stroke patients with unfavorable outcome & 24 & $142.45 \pm 38.7$ \\
\hline
\end{tabular}


Table 5 The relation between risk factors of stroke and copeptin level at presentation

\begin{tabular}{|c|c|c|c|c|c|c|}
\hline \multicolumn{4}{|l|}{ Risk factor } & \multicolumn{3}{|c|}{ Copeptin level at presentation } \\
\hline & & Number & Percent (\%) & Mean & SD & $P$ value \\
\hline \multirow[t]{2}{*}{ Age } & $\leq 45$ & 9 & 20 & 104.81 & 38.85 & 0.102 \\
\hline & $>45$ & 36 & 88 & 109.93 & 36.30 & \\
\hline \multirow[t]{2}{*}{ Sex } & Males & 19 & 42.2 & 106.96 & 39.97 & 0.169 \\
\hline & Females & 26 & 57.8 & 100.39 & 37.9 & \\
\hline \multirow[t]{2}{*}{ Hypertension } & Yes & 13 & 28.9 & 118.89 & 40.4 & 0.05 \\
\hline & No & 32 & 71.1 & 93.84 & 37.25 & \\
\hline \multirow[t]{2}{*}{ Diabetes Mellitus } & Yes & 16 & 35.6 & 119.17 & 48.5 & 0.024 \\
\hline & No & 29 & 64.4 & 92.99 & 43.1 & \\
\hline \multirow[t]{2}{*}{ Dyslipidemia } & Yes & 12 & 26.7 & 123.77 & 46.4 & 0.05 \\
\hline & No & 33 & 73.3 & 93.84 & 44.2 & \\
\hline \multirow[t]{2}{*}{ Cardiac diseases } & Yes & 29 & 64.4 & 112.21 & 39.2 & 0.547 \\
\hline & No & 16 & 35.6 & 119.62 & 40.6 & \\
\hline \multirow[t]{2}{*}{ Carotid stenosis } & $<50 \%$ & 34 & 75.6 & 110.30 & 35.4 & 0.728 \\
\hline & $\geq 50 \%$ & 11 & 24.4 & 119.53 & 38.9 & \\
\hline
\end{tabular}

Table 6 Copeptin level in stroke patients who received rTPA and patients who did not receive rTPA

\begin{tabular}{llll}
\hline & $\begin{array}{l}\text { Patient received } \\
\text { rTPA }(\mathbf{n}=\mathbf{1 1})\end{array}$ & $\begin{array}{l}\text { Patient did not } \\
\text { receive rTPA } \\
\mathbf{( n = 3 4 )}\end{array}$ & P-value \\
\hline $\begin{array}{l}\text { Copeptin }(\mathrm{pmol} / \mathrm{l}) \\
\text { Mean } \pm \text { SD }\end{array}$ & $99.55 \pm 37.6$ & $127.31 \pm 46.4$ & 0.049 \\
\hline
\end{tabular}

Table 7 Correlation between demographic, clinical, laboratory, radiological parameters and copeptin level

\begin{tabular}{lll}
\hline & \multicolumn{2}{l}{ Copeptin level } \\
\cline { 2 - 3 } & $\mathbf{r}^{\mathbf{a}}$ & $\mathbf{p}$-value \\
\hline Age & 0.212 & 0.045 \\
BMI & 0.272 & 0.009 \\
Uric acid & 0.256 & 0.015 \\
Volume of infarction & 0.299 & 0.004 \\
Stroke severity at presentation (meas- & 0.328 & 0.002 \\
ured by NIHSS) & & \\
Clinical outcome after 3 months & 0.378 & 0.001 \\
(measured by mRS) & & \\
\hline BMI body mass index & &
\end{tabular}

As demonstrated in Fig. 1, receiver operating characteristic (ROC) curve analysis was used to assess the prognostic role of copeptin level after acute ischemic stroke. With sensitivity of $62.2 \%$ and specificity of $84.4 \%$, the optimal cutoff value of copeptin that predicted unfavorable stroke outcome was $\geq 125.30 \mathrm{pg} / \mathrm{ml}$

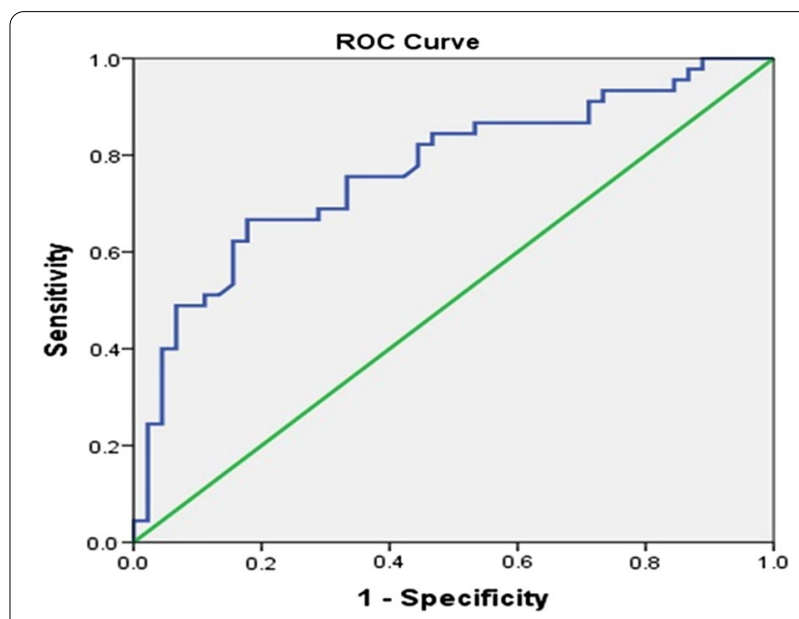

Fig. 1 ROC curve analysis to assess the prognostic role of copeptin level after acute ischemic stroke

(the area under the curve $0.769,95 \%$ confidence interval 0.671 to 0.868 , P-value $=0.012$ )

\section{Discussion}

Copeptin is a new blood biomarker which has emerged to assist clinicians with decision-making. Copeptin can accurately reflect vasopressin concentration in both serum and plasma, in a similar fashion to C-peptide for insulin [10]. Copeptin can detect the extent of threat to each patient and the individual stress response to that threat. Therefore, the elevation of serum copeptin may be an indication that the patient required more attention 
and further evaluation, especially since copeptin level is elevated in life-threatening conditions [11, 12].

We investigated the role of copeptin as a blood biomarker to predict short-term outcome in patients presenting with acute ischemic stroke to the emergency room and outpatient clinic. An early risk assessment with evaluation of disease severity and prognosis is essential for optimum care and allocation of healthcare resources.

Our study was conducted on 45 patients with first ever acute ischemic stroke with symptom onset within $24 \mathrm{~h}$ and 45 control subjects. Serum level of copeptin was measured for both the control and the patients groups at admission.

In this study, serum copeptin levels were much higher in patients with acute ischemic stroke compared to healthy controls. This was in agreement with Zeng and colleagues [11], Dong and colleagues [12], and Maestrini and colleagues [13].

Our findings could be explained by the fact that the elevation of copeptin level reflects the activation of the endogenous stress system; vasopressin is an efficient synergistic factor of corticotrophin-releasing hormone, which stimulates both the synthesis and secretion of adrenocorticotrophic hormone (ACTH) by the anterior pituitary gland [14].

Also, copeptin plays a role in ischemic neuronal injury; as it contributes to $\mathrm{K}$ channel dysfunction after brain injury through a mechanism independent of protein kinase $\mathrm{C}$ activation [15].

AVP stimulates V1a and V2 receptors which trigger platelet aggregation, vasoconstriction, and water retention and as a result, hypovolemic or normovolemic hyponatremia and low plasma osmolality may occur [16].

On the other hand, Wendt and colleagues [17] found no statistically significant difference between stroke patients and control group regarding copeptin level which was measured at different time onset from symptoms. These results may be due to the fact that the early assessment of copeptin level after onset of symptoms provides a better more significant differentiation between acute ischemic stroke patients and the healthy control subjects.

Age is the most powerful non-modifiable risk factor of stroke as the incidence of stroke doubles with every decade over the age of 55 years [18].

In this study, the serum copeptin level has a statistically significant positive correlation with age of the patients. This finding is in agreement with Molnar and colleagues [19] whose data suggested that age could modulate the results of copeptin.

Elderly people had limited physiologic responses to serious illness as acute stroke, at the same time, they had a higher incidence to neuronal ischemia and more prone to life-threatening complications and bad prognosis which was associated with higher copeptin level [20].

On the contrary, Wang and colleagues [21] found that copeptin levels showed no differences between different ages of patients.

Hypertension is a major modifiable risk factor for cerebrovascular disease. The risk of stroke increases proportionately with the increase of blood pressure. Our study demonstrated that copeptin level was significantly higher in patients with hypertension as compared with patients without hypertension. These results are in agreement with Montaner and colleagues [22].

This relation may be explained because the peripheral effects of AVP are exerted by binding to tissue-specific G-protein-coupled receptors (GPCRs) one of them is the $V_{1}$ receptor, which mediates arterial vasoconstriction. Also, hypertension may occur due to catecholamine surge as a part of stress response which occurs after acute ischemic stroke and associated with higher copeptin level [23].

DM is known to cause both microvascular and macrovascular complications, including cerebrovascular disease. In this study, copeptin level was significantly higher in acute stroke patients with DM as compared to patients without DM. this finding was in agreement with Morgenthaler and colleagues [24].

Higher copeptin levels were significantly associated with type 2 diabetes. Copeptin can be used as a novel risk marker for diabetes susceptibility earlier in the prediabetes state [24]. The arginine-vasopressin system is postulated to play a role in glucose metabolism, as high plasma vasopressin was associated with higher incidence of insulin resistance [25].

Plasma copeptin level also showed a positive correlation with dyslipidemia and BMI. This is in agreement with Muller and colleagues [25] and Nickel and colleagues [1] who found that the best correlation with copeptin was reported with high triglycerides and discovered a concordant correlation between higher copeptin levels and metabolic syndrome or its components.

The relation between copeptin and high triglyceride levels can be explained by increased hepatic synthesis of triglycerides due to the effect of glucocorticoids, adrenaline, and glucagon which are considered as stress hormones and are upregulated by AVP. The relation between copeptin and the increase in BMI can be due to the increase in cortisol levels which increases appetite and alters fat distribution in the body [26].

Our study showed no statistically significant difference between stroke patients with cardiac disease and their counterparts who did not have cardiac disease regarding copeptin level. 
This result is in agreement with Wang and colleagues [21]. AVP interacts with two opposing receptors in the heart: activation of the cardiac $\mathrm{P}_{2}$ purinoceptors leads to coronary vasoconstriction and negative inotropy, while stimulation of oxytocin receptors in the heart stimulates the release of atrial natriuretic peptide. So, the net result is a little effect of AVP on the heart [21].

In contrast, Giannopoulos and colleagues [27] found that copeptin can be used as a diagnostic and prognostic blood biomarker for myocardial infarction and acute heart failure. Due to effect of AVP on $V_{1}$ receptors which are also present on cardiac myocytes, but they concluded that the vasoconstrictive effect of AVP on these cells is dose-dependent and still under debate [27].

The current study demonstrated that no statistically significant difference was found between patients with normal carotid duplex and patient with carotid artery stenosis with regard to copeptin level. Our finding is in agreement with Wang and colleagues [21] who found no significant difference in copeptin levels between subjects with normal carotid artery duplex and those who had higher carotid artery intima-media thickness. Unlike El Dayem and colleagues [28], who concluded that copeptin is an inflammatory marker and associated with increase in carotid artery intima-media thickness and can be a predictor for atherosclerosis. The difference between our findings and that of El Dayem and colleagues can be explained by the difference in patient groups, as all the patients included in El Dayem and colleagues study were diabetics, while only $35.6 \%$ of our patients were diabetic, many studies elaborated higher copeptin levels in diabetic patients as discussed before.

Tissue plasminogen activator is the only proved effective medical therapy for acute ischemic stroke treatment to date. Our study demonstrated that copeptin level was significantly lower in patients received rTPA as compared with patients who did not receive rTPA. The lower levels of serum copeptin with intravenous thrombolysis support its role in reducing ischemic lesion volume and neurological after acute stroke [29].

In this study, the National Institutes of Health Stroke Scale (NIHSS) was used to assess stroke severity on admission. We found that serum copeptin level when measured within $24 \mathrm{~h}$ of stroke onset was correlated significantly with stroke severity on admission. This result is in agreement with Zeng and colleagues [11] and Dong and colleagues [12].

Also, Katan and colleagues [2] who conducted a prospective cohort study on 362 consecutive patients with an acute ischemic stroke found that in all patients with an acute ischemic cerebrovascular event, copeptin levels increased with increasing severity of stroke measured by the NIHSS score.
Different mechanisms can explain the increase in copeptin level in severe acute ischemic stroke; vasopressin enhances vasogenic edema and cellular swelling after ischemic insult. Cerebral edema occurs more with severe stroke compared to mild or moderate stroke and may be a major factor in early and remote complications of stroke. Also, major stroke was associated with hyponatremia in about $40 \%$ of the patients in addition to syndrome of inappropriate secretion of antidiuretic hormone and cerebral salt wasting syndrome. Both of hyponatremia and volume overload stimulate the release of vasopressin [11].

In contrast to our results, Reganon and colleagues [30] found that there was no significant correlation between copeptin levels and NIHSS in patients with acute ischemic stroke. This can be explained by their limited number of patients, as they included only 20 stroke patients.

In the present study, serum copeptin has a statistically positive correlation with the initial infarction volume measured on CT brain or MRI brain. This is in agreement with Katan and colleagues [2], Zeng and colleagues [11], Dong and colleagues [12] and Reganon and colleagues [30].

These results could be explained by the close relation between serum copeptin level and cerebral edema which develops early after the onset of focal ischemia and correlates well with the volume of infarction [31].

Vasopressin receptor $\mathrm{V} 1 \mathrm{a}$ is involved in the pathogenesis of secondary brain damage following acute ischemia via increasing cerebral edema. Cerebral edema leads to an increase in brain volume and elevation of intracranial pressure, which has negative impacts on cerebral blood flow [11].

Our study used mRS score to assess functional outcome after 3 months from the onset of stroke symptoms and found that the higher the copeptin levels, the higher the mRS score. Also copeptin level was much higher in stroke patients with unfavorable outcome $(\mathrm{mRS}=3-6)$ than stroke patients with favorable outcome $(\mathrm{mRS}=0-2)$.

In agreement with our findings, Katan and colleagues [2], Zeng and colleagues [11], Dong and colleagues [12] and Maestrini and colleagues [13] found that patients with an unfavorable outcomes and non-survivors had significantly increased copeptin levels on admission and showed that copeptin was an independent predictor of functional outcome and non-survivors.

Also, the results of a meta-analysis included a total of 1976 acute ischemic stroke patients from 6 studies showed that patients with poor outcomes and non-survivors had a higher copeptin level at admission so assessment of copeptin level can help in decision-making for different types of therapeutic interventions [32]. 
The relation between serum copeptin level and bad prognosis after stroke could be explained by the relationship between vasopressin levels and the development of cerebral edema [32]. Blocking of vasopressin receptors decreases brain edema in ischemic and traumatic mice models [33].

In addition, copeptin is significantly increased in bacterial infection and febrile conditions which occur as post-stroke complications and worsen the prognosis of stroke [34].

Furthermore, Deveci and colleagues [35] found that the increase of plasma copeptin level may indicate that the patient needs further evaluation, especially since copeptin level is elevated in life-threatening conditions, such as acute myocardial infarction, shock, heart failure, pneumonia, pulmonary thromboembolism and renal failure.

The main limitation of this study is the relatively small number of the studied groups as this work is selffunded. Further studies which include larger number of patients are needed to verify the results of this study. Also, only a single measurement of serum copeptin was collected, follow-up measurement of copeptin level in different time intervals with correlation with the clinical state of the patients would be more valuable.

\section{Conclusions}

Copeptin appears to have an interesting potential as a new prognostic biomarker for patients with acute ischemic stroke as copeptin level was significantly higher in patients with severe stroke (NIHSS $>16)$ and in patients with unfavorable outcome (mRS 3-6). This may allow better risk assessment and allocation of targeted therapies for stroke patients in the future. However, before wide implementation, cumulative studies are needed for validation. Future research is required to characterize the utility of vasopressin antagonists in treatment of stroke.

\begin{abstract}
Abbreviations
ACTH: Adrenocorticotrophic hormone; AVP: Arginine-vasopressin; BMI: Body mass index; ELISA: Enzyme-linked immunosorbent assays; GPCRs: G-proteincoupled receptors; mRS: Modified Rankin Scale; NIHSS: National Institutes of Health Stroke Scale; TOAST: Trial of Org 10172 in Acute Stroke Treatment.
\end{abstract}

\section{Acknowledgements}

Not applicable.

\section{Authors' contributions}

$\mathrm{MO}$ participated in the study design, collection of data, sequence alignment, and analysis of the data and helped to draft the manuscript. RS participated in the study design, sequence alignment, and analysis of the data and helped to draft manuscript. RA performed the laboratory work and helped to draft the manuscript. AM participated in the study design, collection of the data and helped to draft the manuscript. All authors read and approved the final manuscript.
Funding

The authors did not receive any funding for this work.

\section{Availability of data and materials}

The datasets used and/or analyzed during the current study are available from the corresponding author on reasonable request with the permission of Faculty of Medicine, Beni-Suef University, Egypt.

\section{Declarations}

\section{Ethics approval and consent to participate}

A written informed consent was obtained from each participant in this study or one of his family and the study was approved by the authorized ethical committee in Faculty of medicine, Beni-Suef University (FWA00015574 in 9 Dec, 2018).

\section{Consent for publication}

Not applicable.

\section{Competing interests}

The authors declare that they have no competing interests.

\section{Author details}

${ }^{1}$ Neurology Department, Beni-Suef University, Beni-Suef 62511, Egypt. ${ }^{2}$ Clinical Pathology Department, Beni-Suef University, Beni-Suef 62511, Egypt.

Received: 6 June 2021 Accepted: 8 October 2021

Published online: 20 October 2021

\section{References}

1. Nickel CH, Bingisser R, Morgenthaler NG. The role of copeptin as a diagnostic and prognostic biomarker for risk stratification in the emergency department. BMC Med. 2012;10:7.

2. Katan M, Fluri F, Morgenthaler NG, Schuetz P, Zweifel C, Bingisser R, et al. Copeptin: a novel, independent prognostic marker in patients with ischemic stroke. Ann Neurol. 2009;66(6):799-808.

3. Sultan SM, Schupf N, Dowling MM, Deveber GA, Kirton A, Elkind MS. Review of lipid and lipoprotein(a) abnormalities in childhood arterial ischemic stroke. Int J Stroke. 2014;9(1):79-87.

4. Barreca T, Gandolfo C, Corsini G, Del Sette M, Cataldi A, Rolandi E, et al. Evaluation of the secretory pattern of plasma arginine vasopressin in stroke patients. Cerebrovasc Dis. 2001;11(2):113-8.

5. Chung JW, Park SH, Kim N, Kim WJ, Park JH, Ku Y, et al. Trial of ORG 10172 in Acute Stroke Treatment (TOAST) classification and vascular territory of ischemic stroke lesions diagnosed by diffusion-weighted imaging. J Am Heart Assoc. 2014;3(4): e001119.

6. NIH Stroke Scale. National Institute of Neurological Disorder website.https://www.ninds.nih.gov/sites/default/files/NIH_Stroke_Scale. pdf. Accessed May 2018.

7. Sacco RL, Diener HC, Yusuf S, Cotton D, Ounpuu S, Lawton WA, et al. Aspirin and extended-release dipyridamole versus clopidogrel for recurrent stroke. N Engl J Med. 2008;359(12):1238-51.

8. Bonita R, Beaglehole R. Modification of Rankin Scale: recovery of motor function after stroke. Stroke. 1988;19(12):1497-500.

9. Sims JR, Gharai LR, Schaefer PW, Vangel M, Rosenthal ES, Lev MH, et al. $\mathrm{ABC} / 2$ for rapid clinical estimate of infarct, perfusion, and mismatch volumes. Neurology. 2009;72(24):2104-10.

10. Christ-Crain M, Fenske W. Copeptin in the diagnosis of vasopressindependent disorders of fluid homeostasis. Nat Rev Endocrinol. 2016:12(3):168-76

11. Zeng X, Deng A, Ding Y, Ni Y, Xu T, Liu J, et al. Copeptin and NT-proBNP as prognostic markers for recurrent ischemic cerebrovascular events in ischemic stroke patients. J Am Coll Cardiol. 2016;68(24):2710-1.

12. Dong $X$, Tao DB, Wang YX, Cao H, Xu YS, Wang QY. Plasma copeptin levels in Chinese patients with acute ischemic stroke: a preliminary study. Neurol Sci. 2013:34(9):1591-5. 
13. Maestrini I, Ducroquet A, Moulin S, Leys D, Cordonnier C, Bordet R. Blood biomarkers in the early stage of cerebral ischemia. Rev Neurol (Paris). 2016;172(3):198-219.

14. Lim CT, Khoo B, et al. Normal physiology of ACTH and GH Release in the hypothalamus and anterior pituitary in man. In: Feingold KR, Anawalt B, Boyce A, et al., editors. Endotext. South Dartmouth (MA): MDText.com, Inc.; 2020.

15. Armstead WM. Vasopressin-induced protein kinase C-dependent superoxide generation contributes to ATP-sensitive potassium channel but not calcium-sensitive potassium channel function impairment after brain injury. Stroke. 2001;32(6):1408-14

16. Sposato LA, Cipriano LE, Saposnik G, Ruíz Vargas E, Riccio PM, Hachinski V. Diagnosis of atrial fibrillation after stroke and transient ischaemic attack: a systematic review and meta-analysis. Lancet Neurol. 2015;14(4):377-87.

17. Wendt M, Ebinger M, Kunz A, Rozanski M, Waldschmidt C, Weber JE, et al. Copeptin levels in patients with acute ischemic stroke and stroke mimics. Stroke. 2015;46(9):2426-31.

18. Navis A, Garcia-Santibanez R, Skliut M. Epidemiology and outcomes of ischemic stroke and transient ischemic attack in the adult and geriatric population. J Stroke Cerebrovasc Dis. 2019;28(1):84-9.

19. Molnár AH, Varga C, Berkó A, Rojik I, Párducz A, László F, et al. Inhibitory effect of vasopressin receptor antagonist OPC-31260 on experimental brain oedema induced by global cerebral ischaemia. Acta Neurochir (Wien). 2008;150(3):265-71.

20. Dobsa L, Edozien KC. Copeptin and its potential role in diagnosis and prognosis of various diseases. Biochem Med (Zagreb). 2013;23(2):172-90.

21. Wang CB, Zong M, Lu SQ, Tian Z. Plasma copeptin and functional outcome in patients with ischemic stroke and type 2 diabetes. J Diabetes Complications. 2016;30(8):1532-6.

22. Montaner J, Perea-Gainza M, Delgado P, Ribó M, Chacón P, Rosell A, et al. Etiologic diagnosis of ischemic stroke subtypes with plasma biomarkers. Stroke. 2008;39(8):2280-7.

23. Holmes CL, Landry DW, Granton JT. Science review: vasopressin and the cardiovascular system part 1-receptor physiology. Crit Care. 2003;7(6):427-34.

24. Morgenthaler NG, Struck J, Alonso C, Bergmann A. Assay for the measurement of copeptin, a stable peptide derived from the precursor of vasopressin. Clin Chem. 2006;52(1):112-9.
25. Müller B, Morgenthaler N, Stolz D, Schuetz P, Müller C, Bingisser R, et al. Circulating levels of copeptin, a novel biomarker, in lower respiratory tract infections. Eur J Clin Invest. 2007;37(2):145-52.

26. Markus R, Reutens DC, Kazui S, Read S, Wright P, Pearce DC, et al. Hypoxic tissue in ischaemic stroke: persistence and clinical consequences of spontaneous survival. Brain. 2004;127(Pt 6):1427-36.

27. Giannopoulos G, Deftereos S, Panagopoulou V, Kossyvakis C, Kaoukis A, Bouras $\mathrm{G}$, et al. Copeptin as a biomarker in cardiac disease. Curr Top Med Chem. 2013;13(2):231-40.

28. El Dayem SMA, Battah AA, El Bohy AEM, Yousef RN, Talaat A. Copeptin as a biomarker of atherosclerosis in type 1 diabetic patients. Open Access Maced J Med Sci. 2020;7(23):3975-8

29. Furlanis G, Ajčević M, Buoite Stella A, Cillotto T, Caruso P, et al. Wake-up stroke: thrombolysis reduces ischemic lesion volume and neurological deficit. J Neurol. 2020;267(3):666-73.

30. Reganon E, Vila V, Martínez-Sales V, Vaya A, Lago A, Alonso P, et al. Association between inflammation and hemostatic markers in atherothrombotic stroke. Thromb Res. 2003;112(4):217-21.

31. Choi KS, Kim HJ, Chun HJ, Kim JM, Yi HJ, et al. Prognostic role of copeptin after stroke: a systematic review and meta-analysis of observational studies. Sci Rep. 2015;5:11665.

32. Xu Q, Tian Y, Peng H, Li H. Copeptin as a biomarker for prediction of prognosis of acute ischemic stroke and transient ischemic attack: a metaanalysis. Hypertens Res. 2017:40(5):465-71.

33. Trabold R, Krieg S, Schöller K, Plesnila N. Role of vasopressin V(1a) and $\checkmark 2$ receptors for the development of secondary brain damage after traumatic brain injury in mice. J Neurotrauma. 2008;25(12):1459-65.

34. Jochberger S, Mayr VD, Luckner G, Wenzel V, Ulmer H, Schmid S, et al. Serum vasopressin concentrations in critically ill patients. Crit Care Med. 2006;34(2):293-9.

35. Deveci F, Öner Ö, Telo S, Kırkıl G, Balin M, Kuluöztürk M. Prognostic value of copeptin in patients with acute pulmonary thromboembolism. Clin Respir J. 2019;13(10):630-6.

\section{Publisher's Note}

Springer Nature remains neutral with regard to jurisdictional claims in published maps and institutional affiliations.

\section{Submit your manuscript to a SpringerOpen ${ }^{\odot}$ journal and benefit from:}

- Convenient online submission

- Rigorous peer review

- Open access: articles freely available online

- High visibility within the field

- Retaining the copyright to your article

Submit your next manuscript at $\boldsymbol{\nabla}$ springeropen.com 